\title{
Erratum: A library of ATMO forward model transmission spectra for hot Jupiter exoplanets
}

\author{
by Jayesh M. Goyal, ${ }^{1 \star}$ Nathan Mayne, ${ }^{1}$ David K. Sing, ${ }^{1}$ Benjamin Drummond, ${ }^{1}$ \\ Pascal Tremblin, ${ }^{2}$ David S. Amundsen, ${ }^{3,4}$ Thomas Evans, ${ }^{1}$ Aarynn L. Carter, ${ }^{1}$ \\ Jessica Spake, ${ }^{1}$ Isabelle Baraffe,${ }^{1}$ Nikolay Nikolov, ${ }^{1}$ James Manners, ${ }^{1,5}$ \\ Gilles Chabrier ${ }^{1,6}$ and Eric Hebrard ${ }^{1}$ \\ ${ }^{1}$ Astrophysics Group, School of Physics and Astronomy, University of Exeter, Exeter EX4 4QL, UK \\ ${ }^{2}$ Maison de la Simulation, CEA-CNRS-INRIA-UPS-UVSQ, USR 3441, Centre d'étude de Saclay, F-91191 Gif-Sur-Yvette, France \\ ${ }^{3}$ NASA Goddard Institute for Space Studies, New York, NY 10025, USA \\ ${ }^{4}$ Department of Applied Physics and Applied Mathematics, Columbia University, New York, NY 10025, USA \\ ${ }^{5}$ Met Office, FitzRoy Road, Exeter, EX1 3PB, UK \\ ${ }^{6}$ Ecole Normale Supérieure de Lyon, CRAL, UMR CNRS 5574, F-69364 Lyon Cedex 07, France
}

Key words: errata, addenda - techniques: spectroscopic-planets and satellites: atmospheres - planets and satellites: composition - planets and satellites: gaseous planets.

The paper 'A library of ATMO forward model transmission spectra for hot Jupiter exoplanets' was published in MNRAS 474, 4, 51585185 .

In the original manuscript (Goyal et al. 2018), we presented a grid of forward model transmission spectra for hot Jupiter exoplanets. However, we recently identified an error in the treatment of rainout in our 1D atmosphere model ATMO. The correction of this error led to changes in the equilibrium chemical abundances using rainout condensation and thereby the transmission spectra. We note that this error only affects the online library ${ }^{2,3}$ that includes rainout condensation, the library with local condensation (without rainout) is unaffected. We further note that the gas phase equilibrium scheme used in ATMO has been compared by Drummond et al. (2016) with the analytical schemes of Burrows \& Sharp (1999) and Heng \& Tsai (2016). The gas phase chemistry with and without local condensation has also been verified in Baudino et al. (2017) against the pet i tCODE (Mollière et al. 2015, 2017) and ExO-REM (Baudino et al. 2015) models. Therefore, issues with the previous version of the grid were confined to the implementation of rainout.

\section{THE RAINOUT CORRECTION}

The Gibbs energy minimization (chemical equilibrium) calculation requires elemental abundances as an input, which are defined as

$B_{i}=\frac{n_{i}^{\text {atom }}}{n^{\text {atom }}}$,

where $n_{i}^{\text {atom }}$ is the number density of atoms of the element $i, n^{\text {atom }}$ is the total number density of atoms and $\mathrm{B}_{i}$ is the mole fraction of the input elemental species. Number density is defined as the number of atoms/molecules/ions of a particular element or chemical species per unit volume of the mixture.
However, equilibrium chemical abundances when computed, are the mole fraction of output product species defined as

$f_{j}=\frac{n_{j}}{n}$,

where $\mathrm{f}_{j}$ is the mole fraction of the species $j, \mathrm{n}_{j}$ is the number density of the species and $n$ is the total number density of gas.

The differences in the quantities, the mole fraction of the input elemental species and output product species defined in equation (1) and equation (2), respectively is clarified using the following example. Suppose we have a box containing one molecule of $\mathrm{H}_{2}$ and one atom of $\mathrm{He}$. In this case, the mole fraction of the element species $\mathrm{H}$ and $\mathrm{He}$ are $\mathrm{B}_{\mathrm{H}}=2 / 3$ and $\mathrm{B}_{\mathrm{He}}=1 / 3$, respectively, since there are two atoms of $\mathrm{H}$, one atom of $\mathrm{He}$ and three atoms in total. The mole fraction of the output product species are $\mathrm{f}_{\mathrm{H}_{2}}=1 / 2$ and $\mathrm{f}_{\mathrm{He}}=1 / 2$, since there is one molecule of $\mathrm{H}_{2}$, one atom of $\mathrm{He}$ and two particles (one molecule plus one atom) in total.

The rainout process in the model is treated by depleting the elemental abundances sequestered by the condensate species, from all of the model layers above (i.e toward lower pressure). In the original manuscript (Goyal et al. 2018), this elemental abundance after rainout on a model level $k$ was calculated as

$B_{i}^{k}=B_{i}^{k-1}-\sum_{j} a_{i j} f_{j}^{k-1}$,

for $k \geq 2$, where the sum is over the number of condensed species only. $a_{i j}$ is the number of atoms of element $i$ in species $j$. We note that $B_{i}^{k=1}$ corresponds to initial input elemental abundances. We further note that the model level $k=1$ denotes the 'bottom' model level (i.e the highest pressure/lowest altitude)

Substituting equation (1) and equation (2) into equation (3), we see that the denominators of the two terms on the right in equation (3) are not equivalent. This is because equation (3) is dealing with the change in the mole fractions of the input elemental species. Thus the second term on the right in equation (3) needs to be appropriately scaled so it returns the elemental species mole fraction for each 


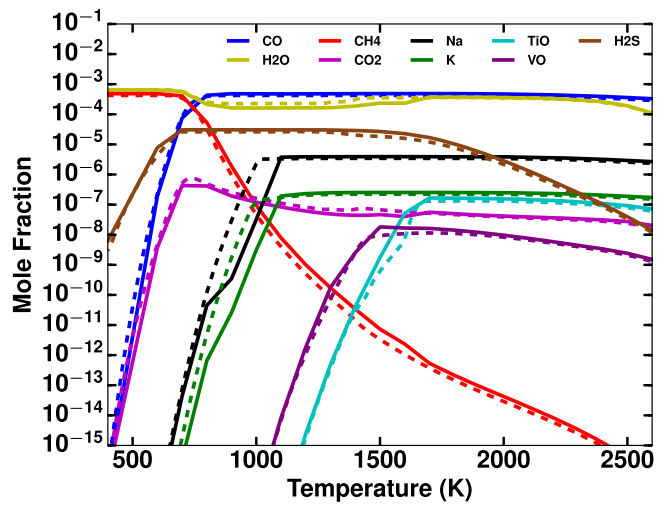

(a)

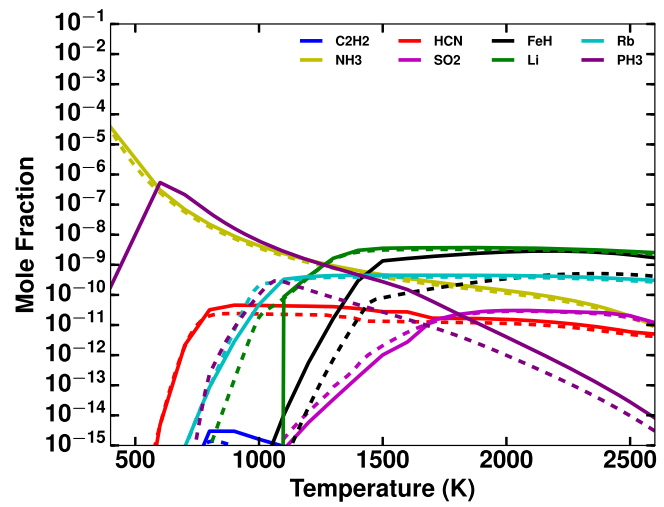

(b)

Figure 1. (a) Figure showing equilibrium chemical abundances from ATMO (solid lines) and GGchem (Woitke et al. 2018) (dashed lines) for a range of temperatures for $\mathrm{CO}, \mathrm{H}_{2} \mathrm{O}, \mathrm{CH}_{4}, \mathrm{CO}_{2}, \mathrm{Na}, \mathrm{K}, \mathrm{TiO}$, $\mathrm{VO}$ and $\mathrm{H}_{2} \mathrm{~S}$ at 1 millibar pressure level in the atmosphere. Horizontal axis shows temperature in $\mathrm{K}$ and vertical axis shows the mole fraction (dimensionless) of the chemical species. (b) Same as 1a but for $\mathrm{C}_{2} \mathrm{H}_{2}, \mathrm{NH}_{3}, \mathrm{HCN}, \mathrm{SO} 2, \mathrm{FeH}, \mathrm{Li}, \mathrm{Rb}$ and $\mathrm{PH}_{3}$.

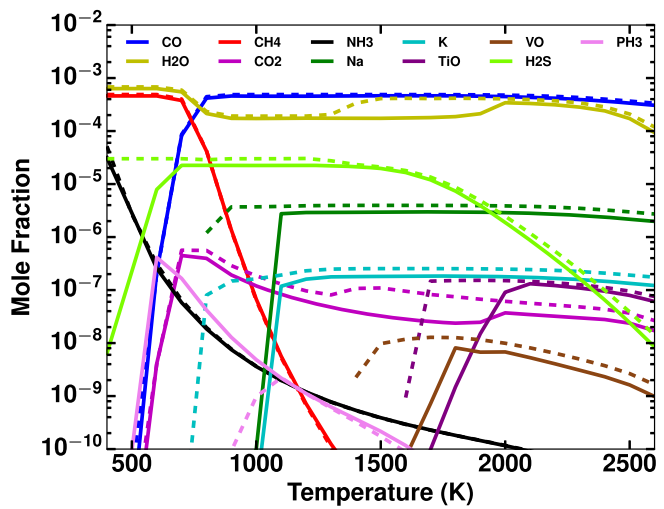

(a)

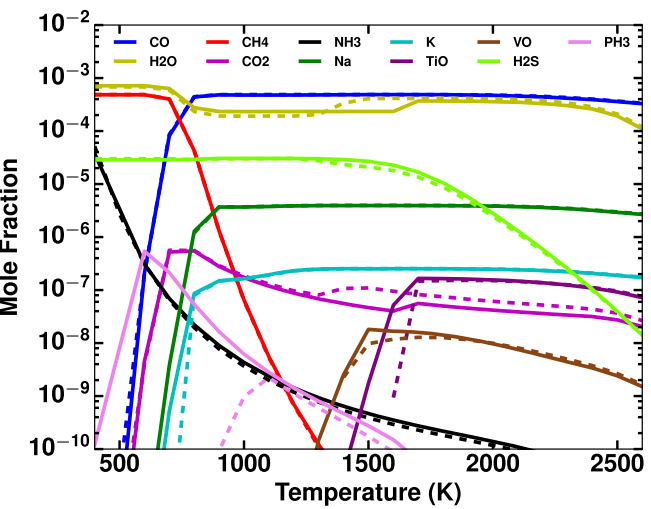

(b)

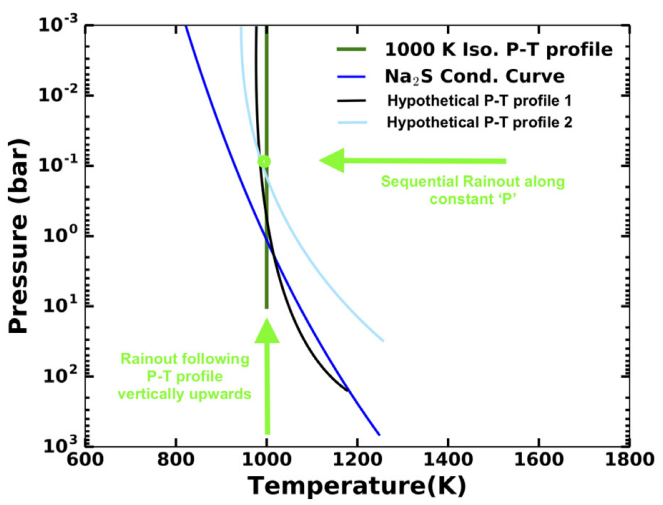

(c)

Figure 2. (a) Figure showing equilibrium chemical abundances with condensation and rainout following the isothermal $P-T$ profile from ATMO (solid lines) with same model inputs as used in the library and Exo-transmit (Kempton et al. 2017) (dashed lines) for a range of temperatures for various important chemical species (in terms of opacity contribution at solar metallicity and solar C/O ratio) at 1 millibar pressure level in the atmosphere. Horizontal axis shows temperature in $\mathrm{K}$ and vertical axis shows the mole fraction (dimensionless) of the chemical species. (b) Same as 2a but with sequential rainout approach along constant pressure (see Section 2 for details) and solar elemental abundances from Lodders (2003) in ATMO. (c) Schematic figure explaining two different rainout techniques, one following the constant temperature from high to low pressure as in ATMO and the other sequential rainout technique, following the constant pressure path from high to low temperature, as adopted in Exotransmit. 


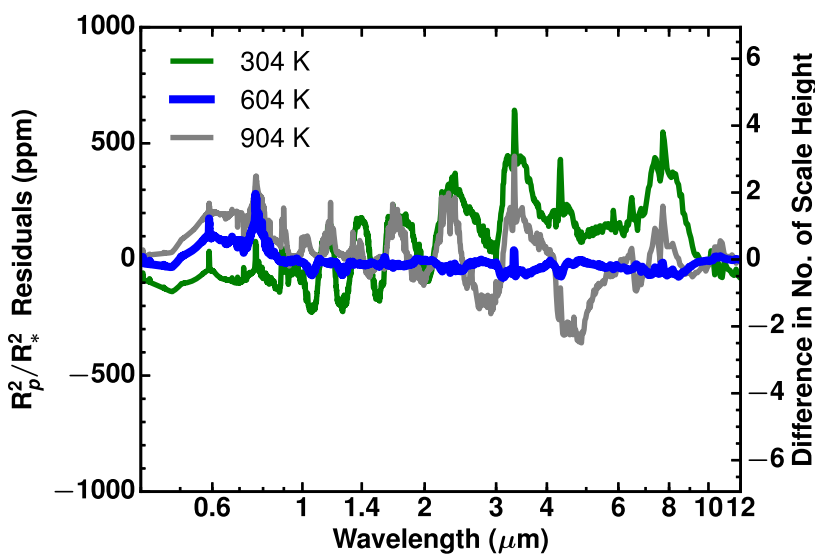

(a)

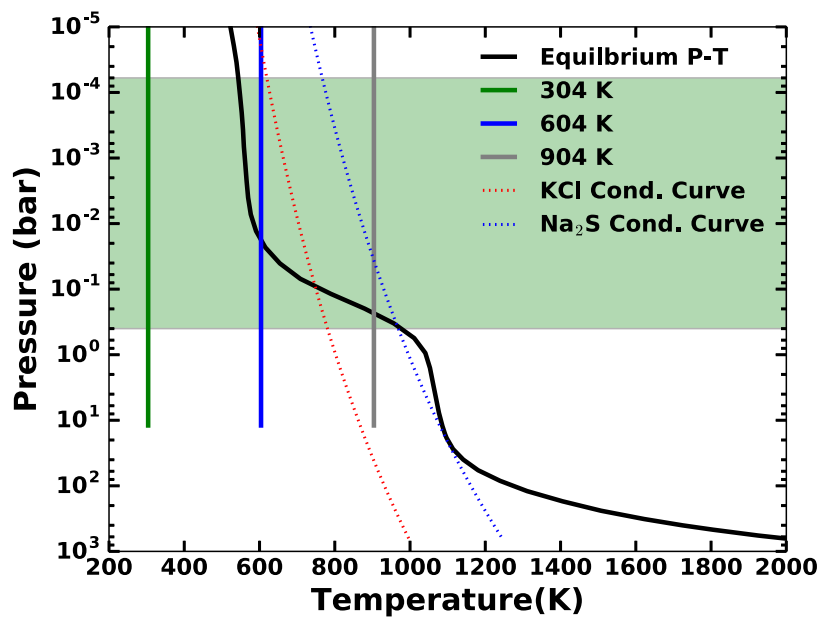

(b)

Figure 3. (a) Figure showing differences (residuals) in transit depth $\left(R_{\mathrm{p}}^{2} / R_{\star}^{2}\right)$ generated using radiative-convective equilibrium $P$ - $T$ profiles and isothermal $P-T$ profile (in the sense consistent minus isothermal) for the isothermal temperatures in our parameter space for GJ $3470 \mathrm{~b}$ which are $304 \mathrm{~K}$ (green), $604 \mathrm{~K}$ (blue) and $904 \mathrm{~K}$ (grey). Thicker line in blue for $604 \mathrm{~K}$ shows minimum residuals and green line for $304 \mathrm{~K}$ shows maximum residuals. Spectra with equilibrium $P-T$ profile is using the recirculation factor of 0.5 . Residuals are shown both in transit depth in parts per million (ppm) on left and number of scale heights on right Y-axis. X-axis shows wavelength in $\mu \mathrm{m}$. (b) Figure showing radiative-convective equilibrium $P$ - $T$ profiles for a recirculation factor of 0.5 (black), and isothermal $P-T$ profiles in our parameter space for GJ $3470 \mathrm{~b}$ which are $304 \mathrm{~K}$ (green), $604 \mathrm{~K}$ (blue) and $904 \mathrm{~K}$ (grey). The condensation curves for KCl and $\mathrm{Na}_{2} \mathrm{~S}$ are also shown with dotted lines in red and blue respectively. Shaded green region highlights the atmospheric pressures (altitude) probed using the transmission spectra. X-axis shows temperature in Kelvin and Y-axis shows pressure in bar. Lower boundary pressure for isothermal $P$ - $T$ profiles has been restricted to 10 bar compared to $10^{3}$ bar adopted earlier in Goyal et al. (2018).

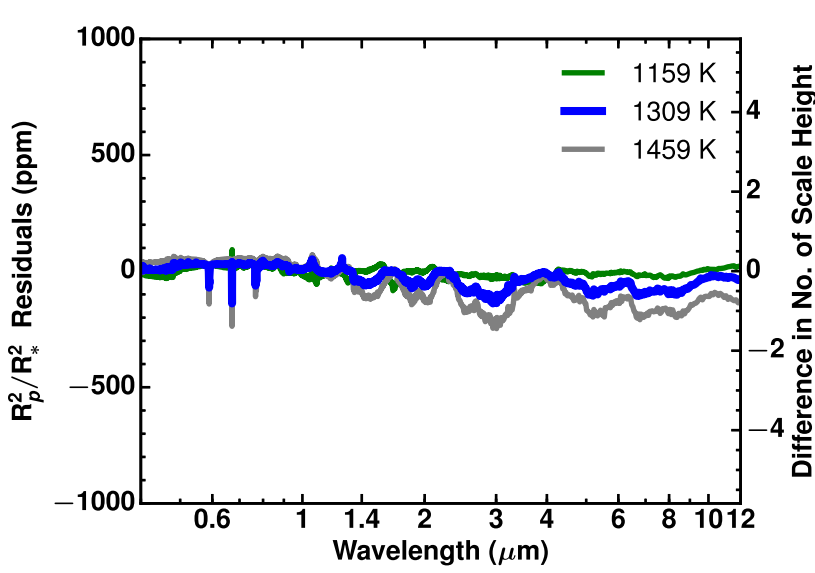

(a)

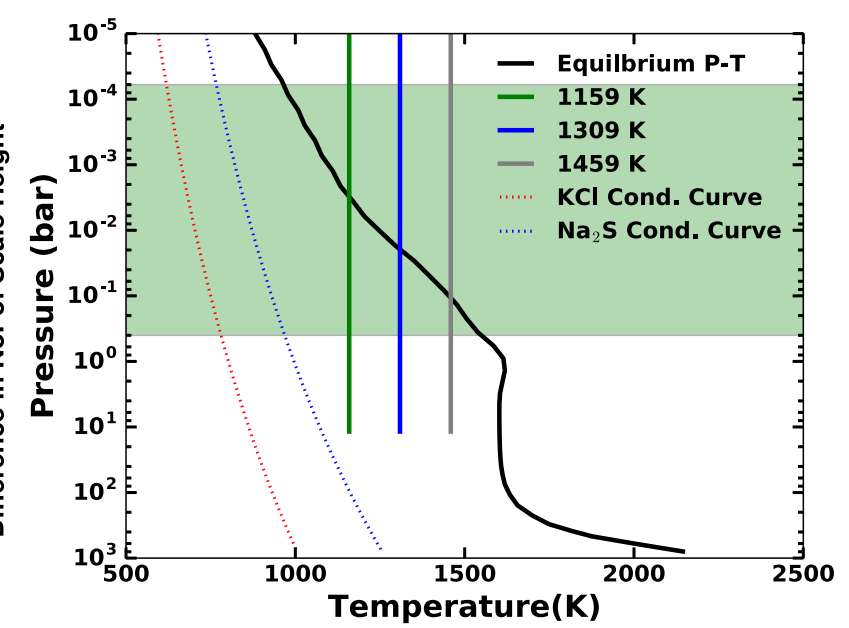

(b)

Figure 4. (a) Figure showing residuals similar to Fig. 3a, but for hotter planet, HD $209458 \mathrm{~b}\left(\mathrm{~T}_{\mathrm{eq}}=1459 \mathrm{~K}\right.$ ) at $1159 \mathrm{~K}$ (green), $1309 \mathrm{~K}$ (blue) and $1459 \mathrm{~K}$ (grey). Thicker line in blue for $1309 \mathrm{~K}$ shows minimum residuals and green line for $1159 \mathrm{~K}$ shows maximum residuals. (b) Figure similar to Fig. $3 \mathrm{~b}$, but for HD 209458 b showing radiative-convective equilibrium $P$ - $T$ profiles for a recirculation factor of 0.5 (black), and isothermal $P$ - $T$ profile at $1159 \mathrm{~K}$ (green), $1309 \mathrm{~K}$ (blue) and $1459 \mathrm{~K}$ (grey).

element and not the mole fraction of the output product species, as was the case in the original manuscript. To correct this we modify equation (3) to

$B_{i}^{k}=B_{i}^{k-1}-\sum_{j} a_{i j} f_{j}^{k-1} \frac{n}{n^{a t o m}}$,

now substituting equation (1) and equation (2) into equation (4) it is clear that the denominators of the two terms are equivalent.
The original, incorrect implementation of the rainout calculation resulted in an erroneously large depletion of the elemental abundances due to condensation, because $n / n^{\text {atom }}$ is typically less than 1 . This most strongly effected the elemental abundance of oxygen, because it is an important element in many condensate species. We have validated the corrected rainout calculation by comparing the initial model input elemental abundances, with the final total element abundances sequestered in various chemical 


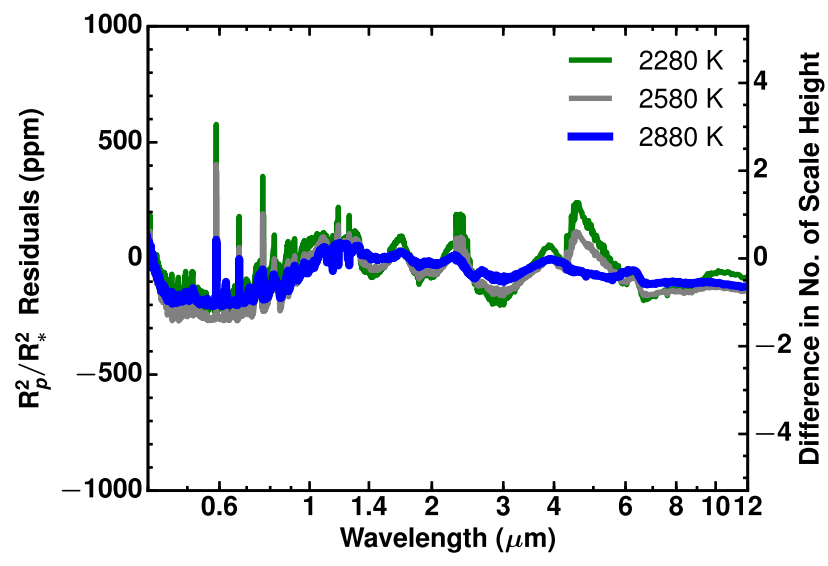

(a)

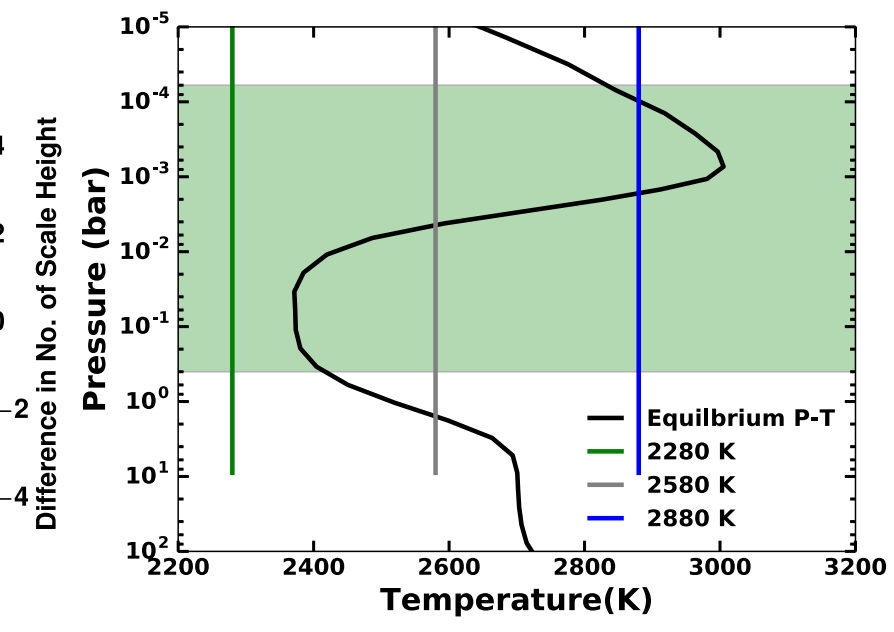

(b)

Figure 5. (a) Figure showing residuals similar to Fig. 3a, but for a hotter planet WASP-12b $\left(\mathrm{T}_{\mathrm{eq}}=2580 \mathrm{~K}\right.$ ) at $2580 \mathrm{~K}$ (green), $2730 \mathrm{~K}$ (grey) and $2880 \mathrm{~K}$ (blue). Thicker line in blue for $2880 \mathrm{~K}$ shows minimum residuals and green line for $2280 \mathrm{~K}$ shows maximum residuals. (b) Figure similar to Fig. $3 \mathrm{~b}$ but for WASP-12 showing radiative-convective equilibrium $P-T$ profiles for a recirculation factor of 0.5 (black), and isothermal $P$ - $T$ profile at $2280 \mathrm{~K}$ (green), $2580 \mathrm{~K}$ (grey) and $2880 \mathrm{~K}$ (blue).

species, which are now conserved. We have also validated this correction by comparing with Exo-transmit model (Kempton et al. 2017), which includes equilibrium condensation with rainout (see Section 2 for details). We note that the bottom of the atmosphere pressure for isothermal pressure-temperature $(P-T)$ profiles is now restricted to 10 bar, in comparison to $10^{3}$ bar adopted in Goyal et al. (2018), since the isothermal $P-T$ profile can be an extreme assumption over a large pressure range, especially with rainout condensation approach (see Goyal et al. 2019, for details).

\section{COMPARISON TO OTHER MODELS}

Fig. 1 presents chemical abundances derived using ATMO (solid lines) compared against those derived using the GGchem chemical model (Woitke et al. 2018), for all the species for which we included opacities in Goyal et al. (2018), except Caesium (Cs), which is not included in GGchem chemical model. For this comparison we adopt local condensation (without rainout) and the solar elemental abundances of Lodders (2003) for both models, and the condensate list for ATMO matches that of Goyal et al. (2018). The major differences between the GGchem and the ATMO model choices used in Goyal et al. (2018) are the included element and condensate species, the methodology to compute equilibrium chemical abundances and the source of thermodynamic data. Fig. 1 shows that the agreement for most of the species is very good, except for a substantial difference in $\mathrm{PH}_{3}$ and to a lesser extent for $\mathrm{FeH}$ and $\mathrm{Li}$. The differences in $\mathrm{PH}_{3}$ abundances, between ATMO and GGchem, is likely due to differences in the list of condensate species included and the source of thermodynamic data. However, doubt on the accuracy of thermodynamic data of various phosphorous species which affect the $\mathrm{PH}_{3}$ abundances, contained in the JANAF database (Chase 1986), has been raised by Lodders \& Fegley (2002), suggesting we cannot perform an accurate benchmarking for this species. Since thermodynamic data for $\mathrm{FeH}$ is not available in the JANAF database, we estimate it using the equilibrium constant for the reaction, $\mathrm{Fe}+\mathrm{H}<=>\mathrm{FeH}$ from Tsuji (1973) in ATMO. However, the
GGChem model adopts the thermodynamic data from Barklem \& Collet (2016), thus offering a potential reason for the differences in $\mathrm{FeH}$. The sudden drop in $\mathrm{Li}$ abundances for temperatures less than $1100 \mathrm{~K}$ in ATMO can be attributed to differences in the condensate list between ATMO and GGchem. However, this is observationally insignificant due to its low abundances. Aside from $\mathrm{PH}_{3}, \mathrm{FeH}$ and Li, Fig. 1 validates the ATMO equilibrium chemistry scheme including local condensation against one of the most up to date and well tested open source chemical equilibrium models available. The detailed comparisons of the equilibrium chemical scheme and predicted abundances of various models is beyond the scope of this erratum.

To validate the ATMO equilibrium chemistry scheme with the assumption of rainout condensation, we compare with the chemical abundances obtained from Exo-transmit (Kempton et al. 2017; Mbarek \& Kempton 2016). Figs $2 \mathrm{a}$ and b, show the equilibrium abundances for the main species (in terms of abundance and opacity contribution at solar metallicity and $\mathrm{C} / \mathrm{O}$ ratio), derived using ATMO and Exo-transmit. For this comparison ATMO has been setup to include condensation with rainout, the solar elemental abundances of Asplund et al. (2009) (as used in Goyal et al. 2018) and the same list of condensates as Goyal et al. (2018). For Exo-transmit, the default values from Lodders (2003) have been used for the solar elemental abundances. Fig. 2a shows a rapid decrease in the abundances of certain species as a function of temperature, caused by condensation and subsequent rainout, at markedly different temperatures between ATMO and Exo-transmit. The main cause of this difference is the numerical technique employed, combined with the assumption of an isothermal temperature-pressure profile. In ATMO the atmospheric chemistry is calculated first at highest pressures, and then following the temperature-pressure profile towards lower pressures. When a given species condenses, the elements that form that condensate are removed from the atmospheric column for all levels at lower pressures, i.e. rainout. However, in Exo-transmit, for a given temperature and pressure, the chemical mixture is calculated by moving, at constant pressure, from $3000 \mathrm{~K}$ toward 


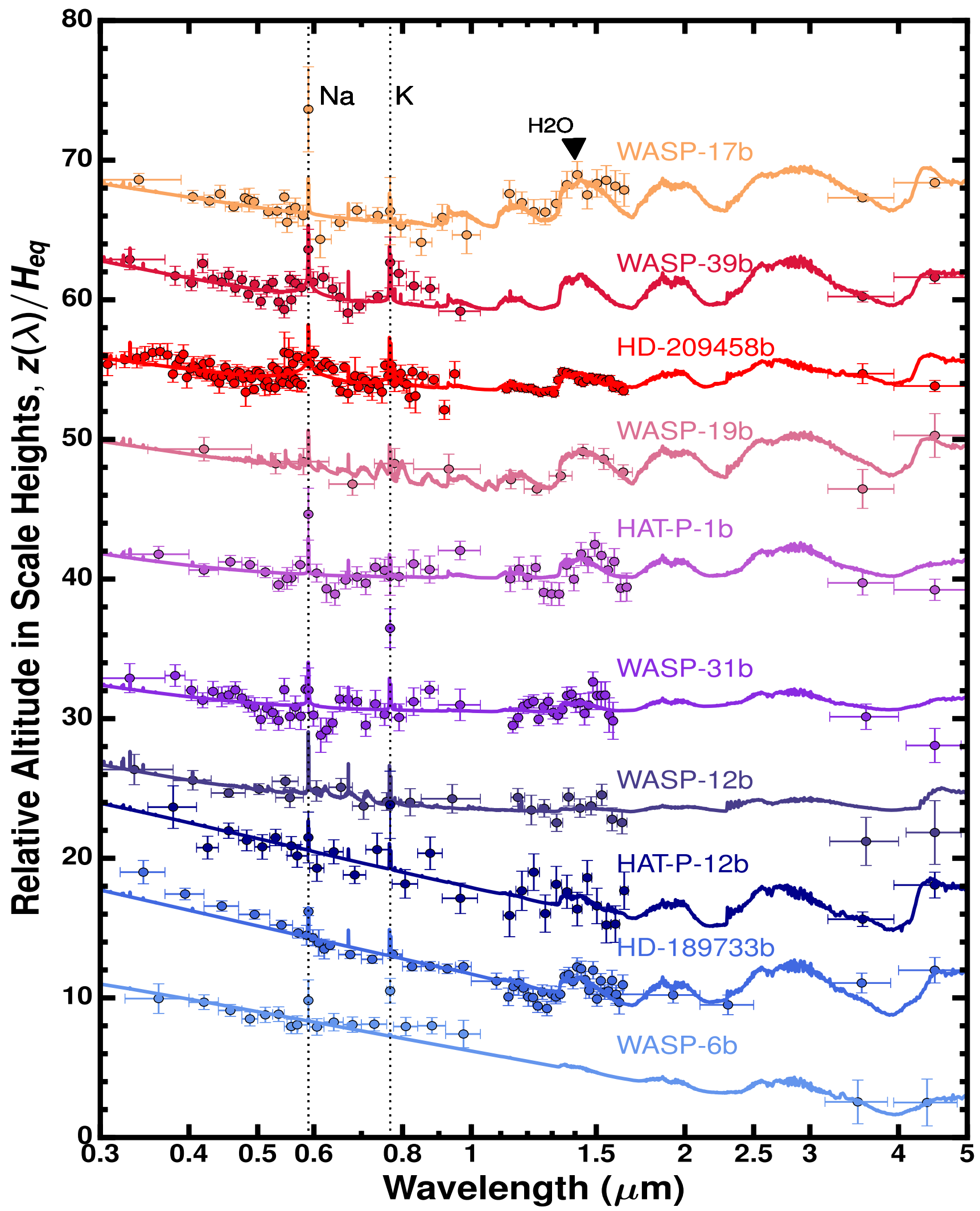

Figure 6. Figure showing the ATMO forward model grid applied to observations of 10 exoplanets from Sing et al. (2016). The Y-axis shows relative altitude in scale height. Solid lines show best fit forward models and filled circular markers show HST observations with error-bars. Planet names are placed above their respective spectra. Dashed lines indicate expected $\mathrm{Na}$ and $\mathrm{K}$ features. Comparatively clear atmospheres at the top have strong $\mathrm{H}_{2} \mathrm{O}$ and alkali features. The strength of these features decreases from top to bottom as planets become more hazy and cloudy. 
Table 1. Table showing best fit planetary characteristics for all the observed exoplanets from Sing et al. (2016). The C/O ratio of 0.56 is solar value. The haze enhancement factor is with respect to gaseous Rayleigh scattering. The grey cloudiness factor is with respect to $\mathrm{H}_{2}$ scattering cross-section at $350 \mathrm{~nm}$. DOF refers to degrees of freedom applied to best fit.

\begin{tabular}{|c|c|c|c|c|c|c|c|c|c|c|}
\hline Planet & $\begin{array}{l}\mathrm{T}_{\mathrm{eq}} \\
(\mathrm{K})\end{array}$ & $\begin{array}{c}\mathrm{T}_{\text {bestfit }} \\
(\mathrm{K})\end{array}$ & $\begin{array}{l}\text { Metallicity } \\
\text { (x solar) }\end{array}$ & $\mathrm{C} / \mathrm{O}$ & $\begin{array}{c}\text { Haze } \\
\left(\alpha_{\text {haze }}\right)\end{array}$ & $\begin{array}{l}\text { Cloud } \\
\left(\alpha_{\text {cloud }}\right)\end{array}$ & $\chi^{2}$ & DOF & Reduced $\chi^{2}$ & Data Source \\
\hline WASP-17b & 1755 & 1605 & 10 & 0.35 & 150 & 1.0 & 32.44 & 38 & 0.853 & Sing et al. (2016) \\
\hline HD-209458b & 1459 & 1159 & 1.0 & 0.7 & 10 & 0.2 & 210.47 & 123 & 1.711 & Sing et al. (2016) \\
\hline WASP-19b & 2077 & 1777 & 10 & 0.56 & 150 & 0 & 7.79 & 13 & 0.60 & $\begin{array}{l}\text { Huitson et al. (2013); } \\
\text { Sing et al. (2016) }\end{array}$ \\
\hline WASP-31b & 1575 & 1425 & 0.005 & 0.35 & 1 & 0.06 & 82.89 & 60 & 1.38 & Sing et al. $(2015,2016)$ \\
\hline WASP-12b & 2580 & 2880 & 0.1 & 0.56 & 150 & 1 & 21.35 & 23 & 0.928 & Sing et al. $(2013,2016)$ \\
\hline HAT-P-12b & 960 & 1260 & 10 & 0.7 & 1100 & 0.2 & 27.25 & 30 & 0.908 & Sing et al. (2016) \\
\hline HD-189733b & 1191 & 1491 & 0.1 & 0.56 & 150 & 0 & 85.88 & 52 & 1.65 & $\begin{array}{l}\text { Pont et al. (2013); } \\
\text { McCullough et al. } \\
\text { (2014); Sing et al. } \\
\text { (2016) }\end{array}$ \\
\hline
\end{tabular}

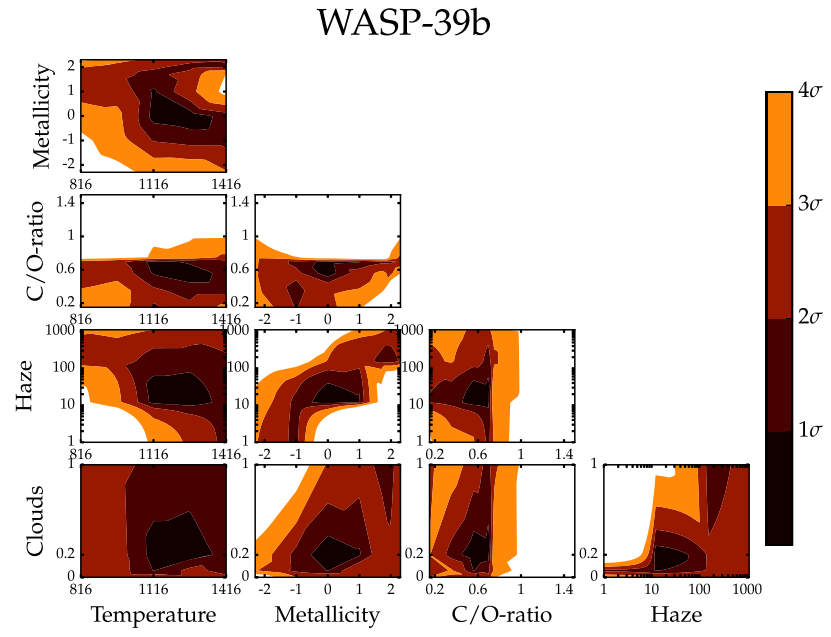

Figure 7. Figure showing $\chi^{2}$ map for WASP-39b. Contours of $\chi^{2}$ are shown for all the combinations of grid parameters. Axis for cloud and haze factors are log-scaled. Metallicity is also log-scaled, 0 being solar metallicity and 2 being 100 times solar metallicity. Colours indicate confidence intervals as shown in colormap to the right.

the required temperature. Similarly, once a condensate is formed it is removed from the atmosphere for subsequent steps. Fig. $2 b$ shows a comparison where the ATMO calculation has been adapted to replicate the rainout technique adopted in Exo-transmit, alongwith the use of solar elemental abundances from Lodders (2003) matching Exo-transmit. The very good agreement in chemical abundances, for almost all species, seen in Fig. $2 b$ reveals that the differences apparent in Fig. 2a are primarily due to model choices. The differences in $\mathrm{PH}_{3}$ abundances seen here can again be attributed to differences in list of condensate species and thermodynamic data, as seen previously when comparing with the GGchem model.

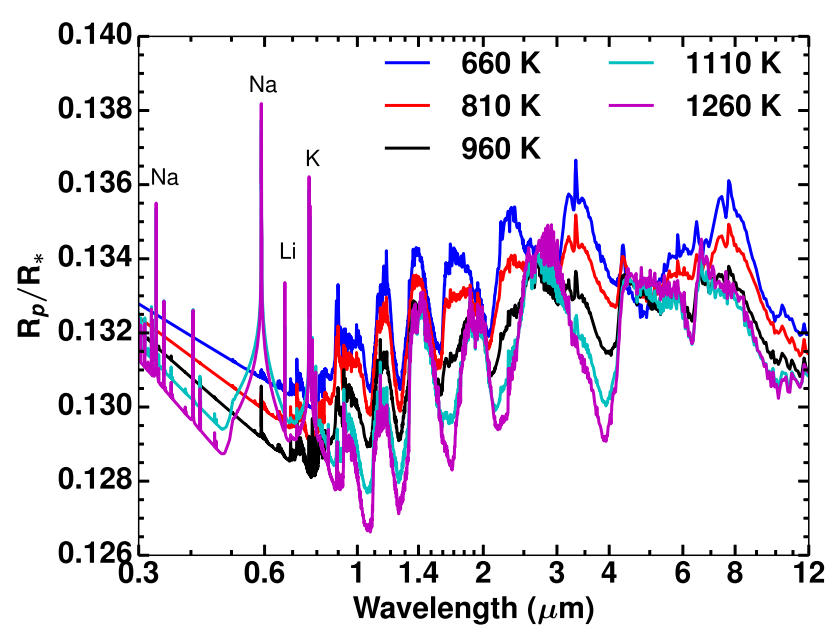

Figure 8. Figure showing HAT-P-12b transmission spectra for a range of temperatures (in Kelvin) at solar metallicity, solar $\mathrm{C} / \mathrm{O}$ ratio and clear atmosphere. $\mathrm{X}$-axis is wavelength in $\mu \mathrm{m}$ and $\mathrm{Y}$-axis transit radius ratio $\left(R_{\mathrm{p}} / R_{\star}\right)$.

The two different rainout approaches adopted by ATMO and Exotransmit, are shown in schematic form in Fig. 2c. Fig. 2c shows the condensation curve for $\mathrm{Na}_{2} \mathrm{~S}$ species, and two hypothetical $P-T$ profiles, alongside an isothermal one. The chemical mixture at the point marked by the green dot, on the isothermal profile (a simplified assumption for two hypothetical $P-T$ profiles) is dependent on the method employed. In ATMO, we follow the isothermal atmospheric $P-T$ profile vertically (i.e. from high to low pressures), performing sequential condensation and rainout to reach the green dot, while Exo-transmit iterates from high to low temperatures, at constant pressure, sequentially condensing species (and raining them out) along this path to reach the green dot. For the first hypothetical 


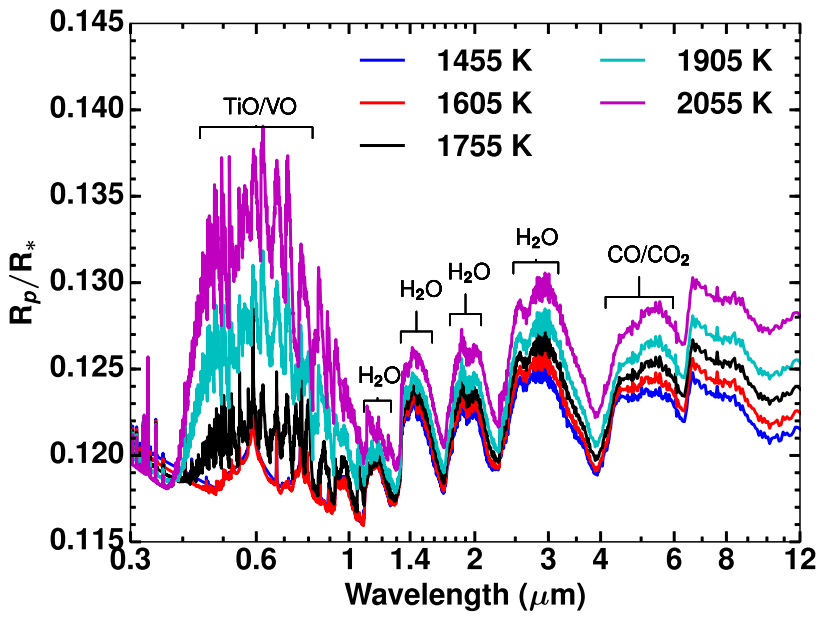

(a)

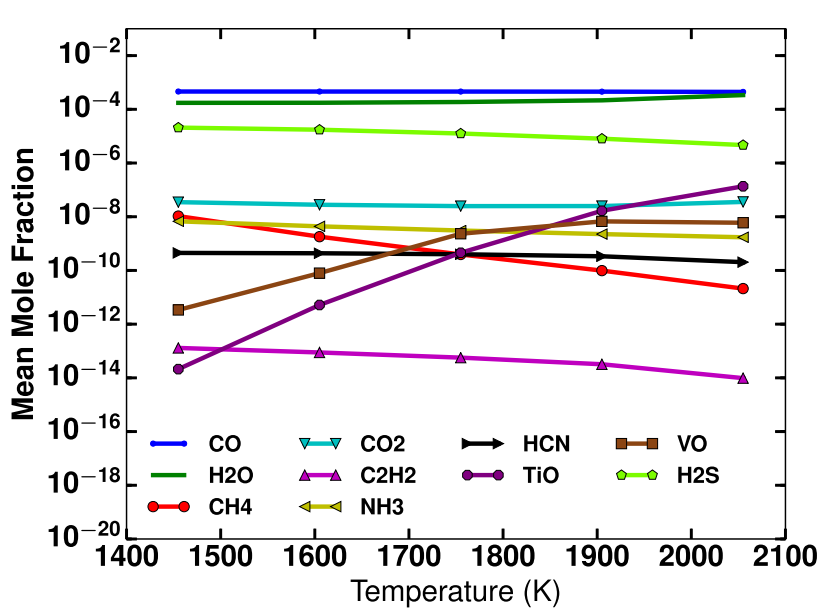

(b)

Figure 9. (a) Figure showing WASP-17b transmission spectra for a range of temperatures, similar to Fig. 8, with major molecular features shown at equilibrium temperature (1755 K). (b) Figure showing change in mean chemical abundances between 0.1 and 100 millibar for various molecules, with change in temperature for WASP-17b at solar metallicity, solar C/O ratio and clear atmosphere. X-axis is temperature in Kelvin while Y-axis shows mean abundances in units of mole fraction.

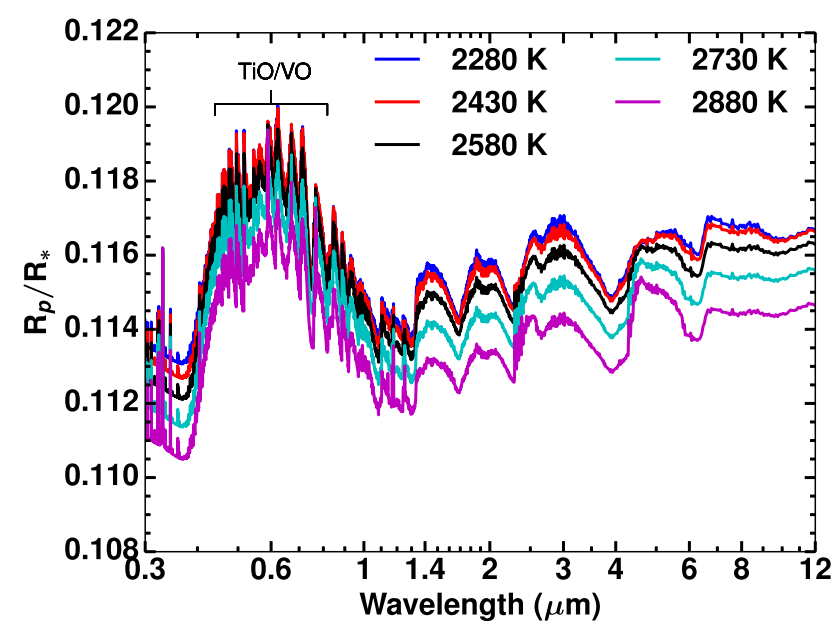

Figure 10. Figure showing WASP-12b transmission spectra for a range of temperatures, similar to Fig. 8.

profile, which crosses the $\mathrm{Na}_{2} \mathrm{~S}$ curve ${ }^{1}$ at high pressures, the ATMO approach will result in a closer agreement between the isothermal and first hypothetical $P$-T profile, whereas the Exo-transmit method will be in disagreement. For the second hypothetical $P-T$ profile, which does not cross the $\mathrm{Na}_{2} \mathrm{~S}$ curve, the Exo-transmit approach will result in a better match between the isothermal and hypothetical $P-T$ profile, under the assumption of rainout.

In summary, the differences in the calculated chemical abundances between ATMO and Exo-transmit can be largely explained by both a choice of condensation/rainout approach and adopted solar elemental abundances. However, neither model approach to condensation is demonstrably more accurate, and as shown schematically in Fig. 2c both can lead to errors under the assumption of an isothermal profile. Therefore, for the purposes of our model grid, as presented here and in Goyal et al. (2018) we retain the

${ }^{1}$ The condensation in both models is calculated using Gibbs energy minimization, so the condensation curves are purely illustrative approach formulated in ATMO, as well as our chosen solar elemental abundances.

\section{REPLACEMENT FIGURES}

All the changed figures are numbered the same as in the original manuscript (Goyal et al. 2018) for ease of comparison. The captions have been slightly updated.

\section{MAJOR CHANGED CONCLUSIONS}

(i) The $\mathrm{H}_{2} \mathrm{O}$ features in the transmission spectra are now larger compared to the results presented in the original manuscript (Goyal et al. 2018), since there is less oxygen depletion (therefore more $\mathrm{H}_{2} \mathrm{O}$ ) with the rainout condensation approach, after the correction of the error. This can be seen in Fig. 6, Figs 8 to 18 and Fig. A10a.

(ii) The models best fitting to observations have been changed slightly as shown in Fig. 6 and Table 1. This has also led to small changes in $\chi^{2}$ maps as shown in Fig. 7 and Figs A1 to A9. The best fit $\mathrm{C} / \mathrm{O}$ ratio for all the planets remain less than or equal to the solar value (0.56), except for HD 209458b and HAT-P-12b where it is 0.7 , slightly higher than the solar value.

(iii) The drastic change in spectral features with change in metallicity from 10 to 50 times solar metallicity due to $\mathrm{HCN}$ and $\mathrm{C}_{2} \mathrm{H}_{2}$ features (seen in figures 11 to 13 in Goyal et al. 2018) is now absent, due to increased availability of oxygen which favours $\mathrm{H}_{2} \mathrm{O}$ formation at these metallicities, as seen in Figs 11 to 13 . Therefore, $\mathrm{HCN}$ and $\mathrm{C}_{2} \mathrm{H}_{2}$ features cannot be used to constrain the metallicities of exoplanet atmospheres as previously concluded, but can be used to constrain $\mathrm{C} / \mathrm{O}$ ratios. The changes in spectral features with increasing metallicity seen in the infrared is due to the increase in $\mathrm{CO}_{2}$ abundances, again due to the availability of more oxygen after the error correction.

(iv) The presence of $\mathrm{TiO} / \mathrm{VO}$ features in the transmission spectrum (with rainout condensation) has now shifted from values greater than $\sim 2000 \mathrm{~K}$ to values greater than $\sim 1700 \mathrm{~K}$, as seen in Fig. 9a. The sudden absence of $\mathrm{TiO} / \mathrm{VO}$ at certain temperatures due 


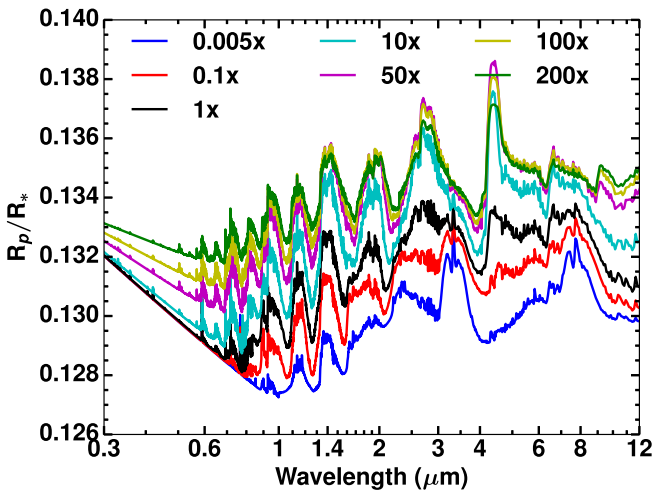

(a)

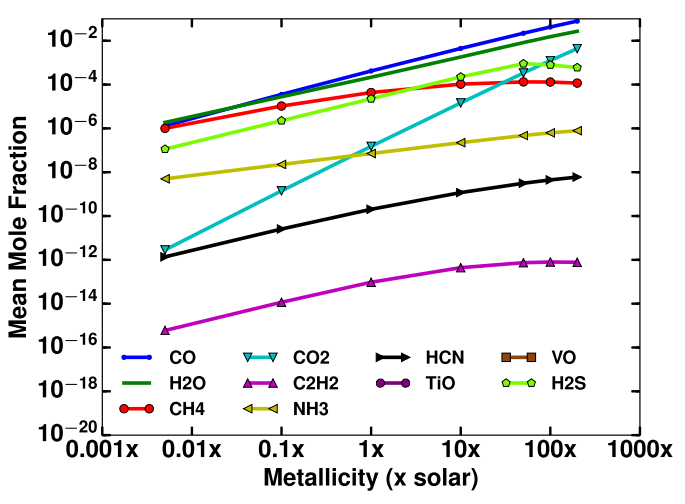

(b)

Figure 11. (a) Figure showing HAT-P-12b transmission spectra for a range of metallicity (times solar) at its equilibrium temperature, solar C/O ratio and clear atmosphere. $\mathrm{X}$-axis is wavelength in $\mu \mathrm{m}$ and Y-axis transit radius ratio $\left(R_{\mathrm{p}} / R_{\star}\right)$. (b) Figure showing change in mean chemical abundances between 0.1 and 100 millibar for various molecules, with change in metallicity for HAT-P-12b. X-axis is metallicity $(\times$ solar) while Y-axis shows mean mole fraction.

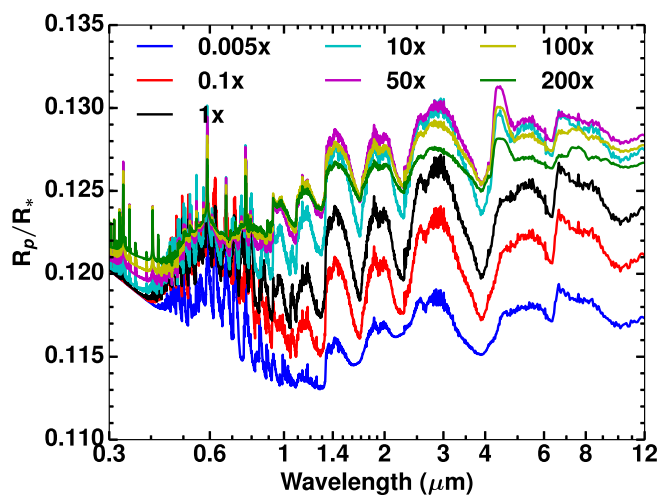

(a)

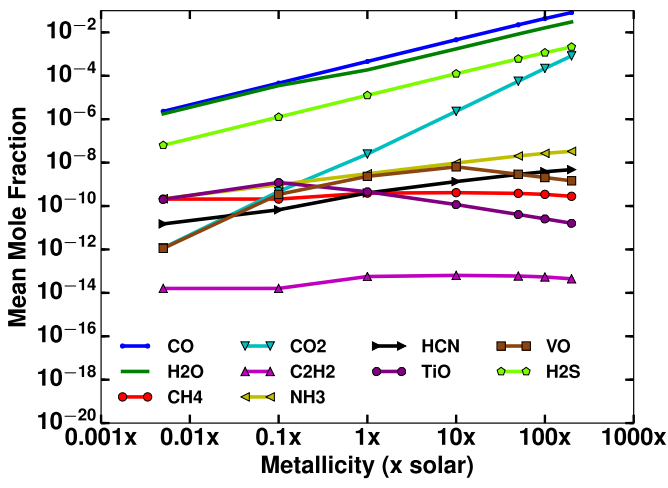

(b)

Figure 12. (a) Figure showing WASP-17b transmission spectra for a range of metallicity (times solar), similar to Fig. 11a, with major molecular features shown at highest metallicity (200x). (b) Figure showing change in mean chemical abundances between 0.1 and 100 millibar for various molecules, with change in metallicity for WASP-17b, similar to Fig. 11b.

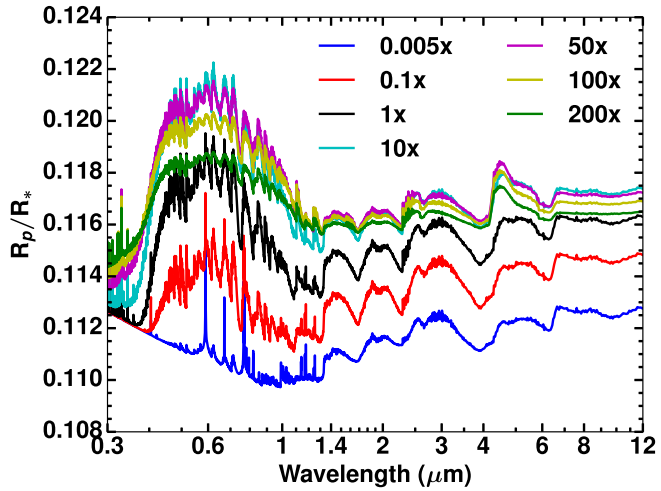

(a)

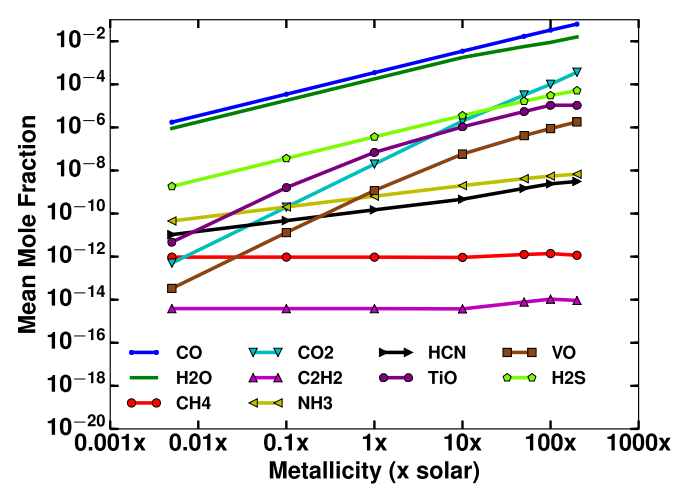

(b)

Figure 13. (a) Figure showing WASP-12b transmission spectra for a range of metallicity (times solar), similar to Fig. 11a. (b) Figure showing change in mean chemical abundances between 0.1 and 100 millibar for various molecules, with change in metallicity for WASP-12b, similar to Fig. 11b. 


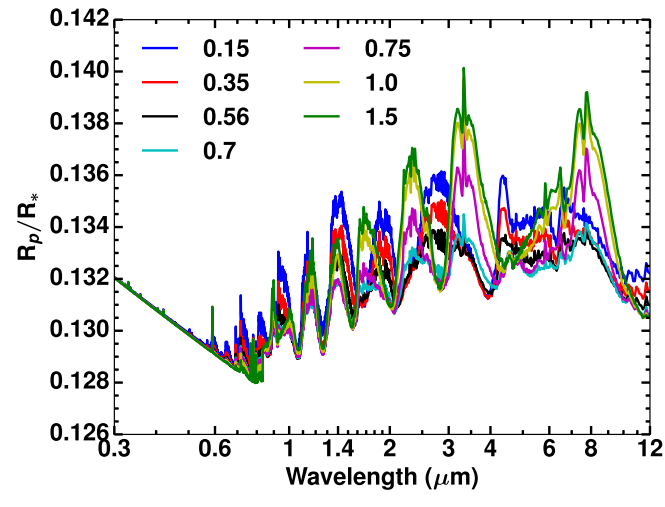

(a)

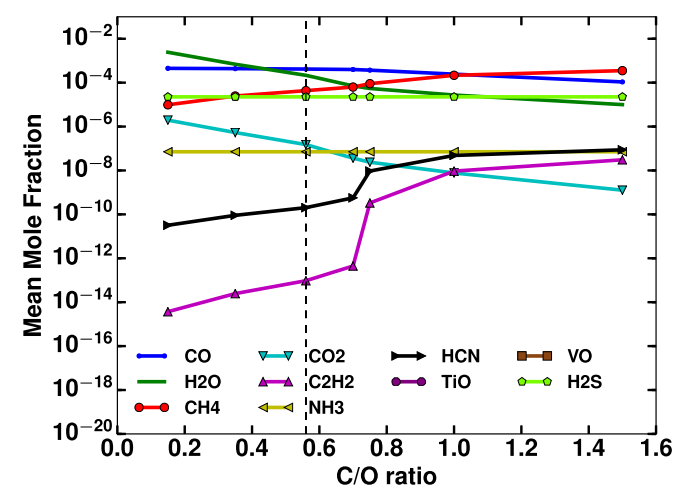

(b)

Figure 14. (a) Figure showing HAT-P-12b transmission spectra for a range of $\mathrm{C} / \mathrm{O}$ ratio at its equilibrium temperature, solar metallicity and clear atmosphere. $\mathrm{X}$-axis is wavelength in $\mu \mathrm{m}$ and $\mathrm{Y}$-axis transit radius ratio $\left(R_{\mathrm{p}} / R_{\star}\right)$. (b) Figure showing change in mean chemical abundances between 0.1 and 100 millibar for various molecules, with change in $\mathrm{C} / \mathrm{O}$ ratio for HAT-P-12b, X-axis is C/O ratio and Y-axis is mean abundances in units of mole fraction. Dashed line indicates solar $\mathrm{C} / \mathrm{O}$ ratio.

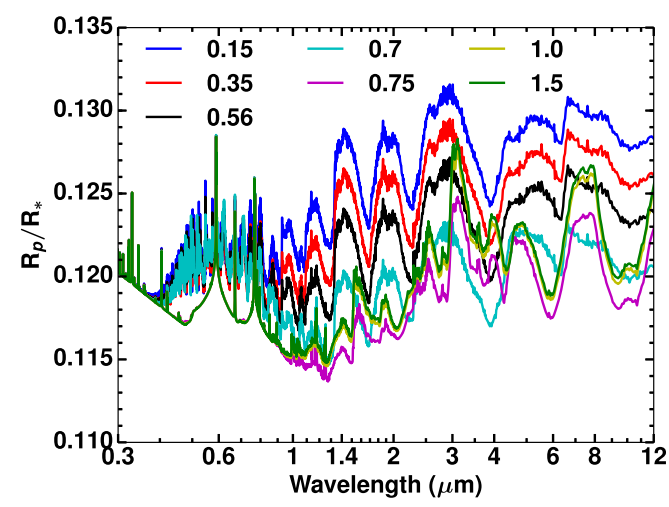

(a)

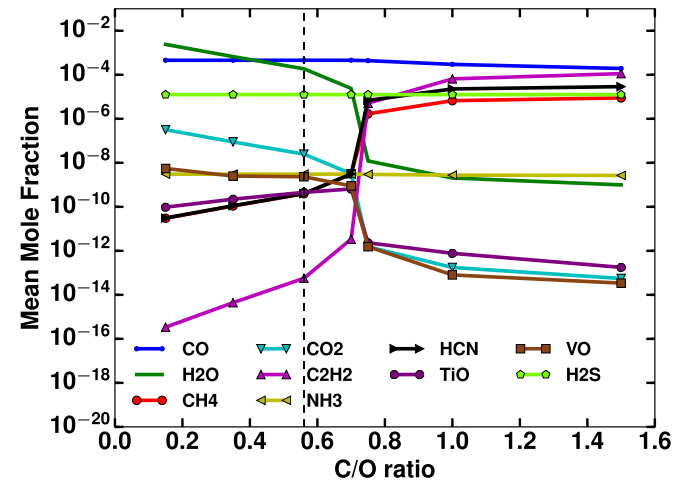

(b)

Figure 15. (a) Figure showing WASP-17b transmission spectra for a range of C/O ratio, similar to Fig. 14a. (b) Figure showing change in mean chemical abundances between 0.1 and 100 millibar for various molecules, with change in C/O ratio for WASP-17b, similar to Fig. 14b.

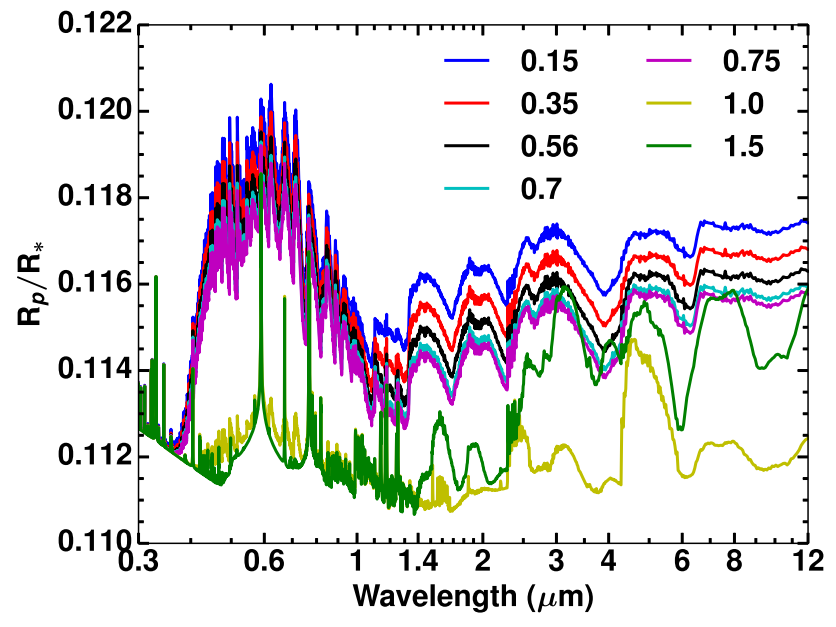

(a)

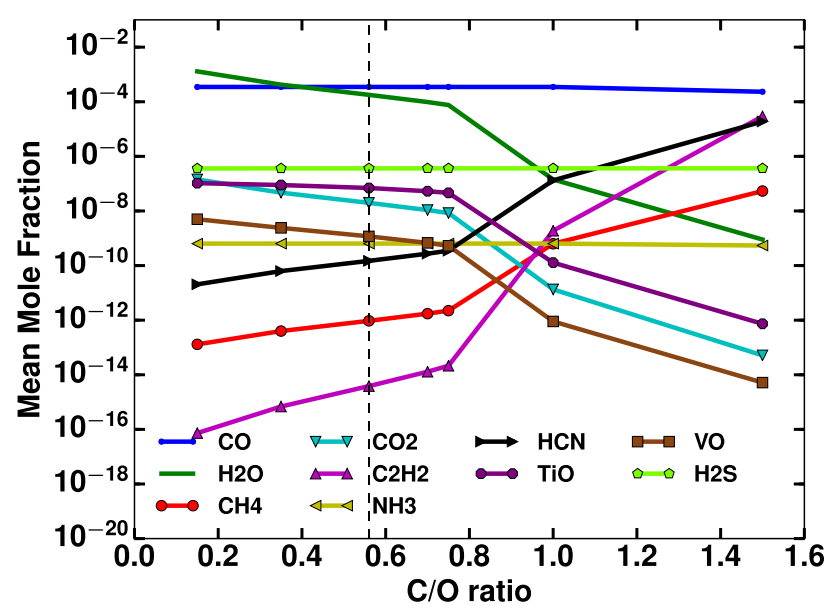

(b)

Figure 16. (a) Figure showing WASP-12b transmission spectra for a range of C/O ratio, similar to 14a. (b) Figure showing change in mean chemical abundances between 0.1 and 100 millibar for various molecules, with change in C/O ratio for WASP-12b, similar to Fig. 14b. 


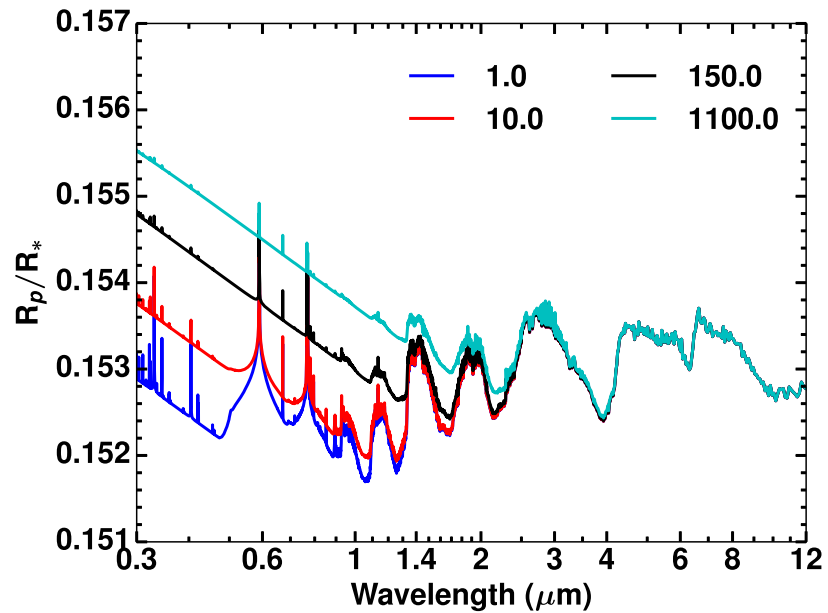

(a)

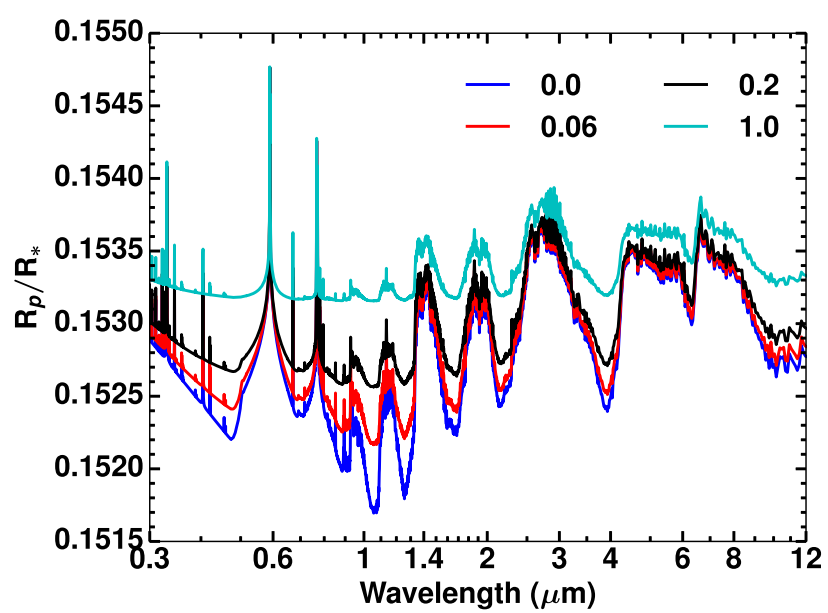

(b)

Figure 17. (a) Figure showing HD $189733 \mathrm{~b}$ transmission spectra for a range of haze enhancement factor at its equilibrium temperature, solar C/O ratio, solar metallicity and no clouds. $\mathrm{X}$-axis is wavelength in $\mu \mathrm{m}$ and $\mathrm{Y}$-axis transit radius ratio $\left(R_{\mathrm{p}} / R_{\star}\right)$. (b) Figure showing HD 189733b transmission spectra for a range of grey cloud enhancement factor at its equilibrium temperature, solar $\mathrm{C} / \mathrm{O}$ ratio, solar metallicity and no haze. $\mathrm{X}$-axis is wavelength in $\mu \mathrm{m}$ and $\mathrm{Y}$-axis transit radius ratio $\left(R_{\mathrm{p}} / R_{\star}\right)$.

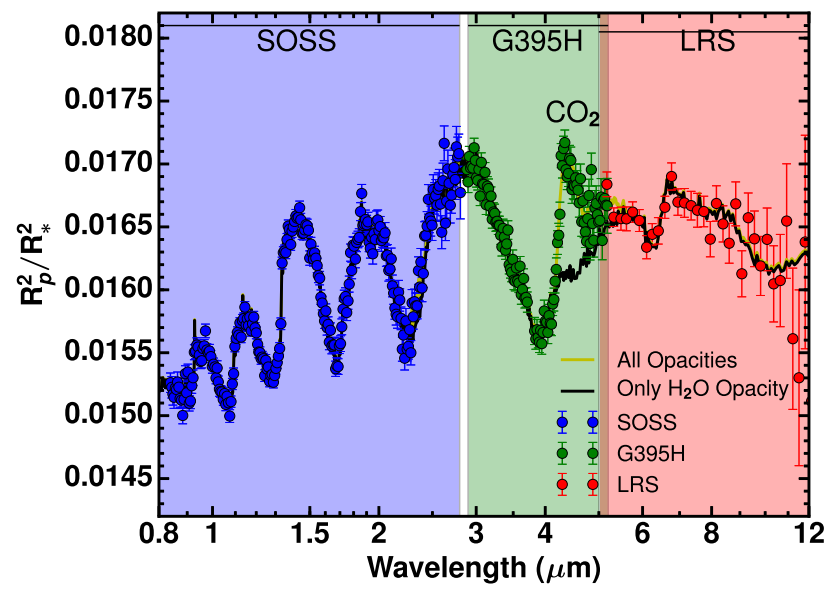

Figure 18. Figure showing ATMO best fit model transmission spectrum (transit depth) for WASP-17b simulated with PandExo for JWST observations. Model spectrum with all opacities is shown in yellow, which for most of the spectrum is hidden behind only $\mathrm{H}_{2} \mathrm{O}$ opacity spectrum shown in black. $\mathrm{CO}_{2}$ (carbon dioxide) feature is marked. Shaded regions and corresponding coloured markers indicate different JWST instrument modes, red indicates NIRISS SOSS mode, blue indicates NIRSpec G395H mode and green indicates MIRI LRS mode. X-axis is wavelength in $\mu \mathrm{m}$ and Y-axis transit depth $\left(R_{\mathrm{p}}^{2} / R_{\star}^{2}\right)$.

to rainout is now a gradual decline in their abundances as seen in Fig. $12 b$.

(v) The best fit model spectrum of WASP-17b with HST observations, when simulated with James Webb Space Telescope (JWST) simulator PandExo now predicts a detectable $\mathrm{CO}_{2}$ feature as seen in Fig. 18, compared to CO feature in (Goyal et al. 2018).

(vi) Restricting lower atmospheric boundary pressure for isothermal $P$ - $T$ profiles to 10 bar, resulted in decrease in transit depth residuals as seen in Figs 3a, 4a and 5a. (vii) The online $\mathrm{e}^{2,3}$ transmission spectra library has been updated with the corrected version.

\section{ACKNOWLEDGEMENTS}

We would like to thank the anonymous reviewer for their constructive comments that improved the erratum. We would like to thank Hannah Wakeford, Mark Marley, Nikole Lewis and Channon Visscher for extensive discussions and model comparisons on the rainout condensation approach that led to the identification of the error.

\section{REFERENCES}

Asplund M., Grevesse N., Sauval A. J., Scott P., 2009, ARA\&A, 47, 481 Barklem P. S., Collet R., 2016, A\&A, 588, A96

Baudino J.-L., Bézard B., Boccaletti A., Bonnefoy M., Lagrange A.-M., Galicher R., 2015, A\&A, 582, A83

Baudino J.-L., Mollière P., Venot O., Tremblin P., Bézard B., Lagage P.-O., 2017, ApJ, 850, 150

Burrows A., Sharp C. M., 1999, ApJ, 512, 843

Chase M. W., 1986, JANAF thermochemical tables. Available at: http://ad sabs.harvard.edu/abs/1986jtt..book.....C

Drummond B., Tremblin P., Baraffe I., Amundsen D. S., Mayne N. J., Venot O., Goyal J., 2016, A\&A, 594, A69

Fischer P. D. et al., 2016, ApJ, 827, 19

Goyal J. M. et al., 2018, MNRAS, 474, 5158

Goyal J. M., Wakeford H. R., Mayne N. J., Lewis N. K., Drummond B., Sing D. K., 2019, MNRAS, 482, 4503

Heng K., Tsai S.-M., 2016, ApJ, 829, 104

Huitson C. M. et al., 2013, MNRAS, 434, 3252

Kempton E. M.-R., Lupu R., Owusu-Asare A., Slough P., Cale B., 2017, PASP, 129, 044402

Lodders K., 2003, ApJ, 591, 1220

${ }^{2}$ http://bd-server.astro.ex.ac.uk/exoplanets/

${ }^{3}$ https://drive.google.com/drive/folders/1Yz94usAAiXtnLR0yoq-qkuhegR rI4u4B 
Lodders K., Fegley B., 2002, Icarus, 155, 393

Mbarek R., Kempton E. M.-R., 2016, ApJ, 827, 121

McCullough P. R., Crouzet N., Deming D., Madhusudhan N., 2014, ApJ, 791, 55

Mollière P., van Boekel R., Dullemond C., Henning T., Mordasini C., 2015, ApJ, 813, 47

Mollière P., van Boekel R., Bouwman J., Henning T., Lagage P.-O., Min M., 2017, A\&A, 605, C3

Nikolov N. et al., 2014, MNRAS, 437, 46

Nikolov N. et al., 2015, MNRAS, 447, 463

Pont F., Sing D. K., Gibson N. P., Aigrain S., Henry G., Husnoo N., 2013, MNRAS, 432, 2917

Sing D. K. et al., 2013, MNRAS, 436, 2956

Sing D. K. et al., 2015, MNRAS, 446, 2428

Sing D. K. et al., 2016, Nature, 529, 59

Tsuji T., 1973, A\&A, 23, 411

Wakeford H. R. et al., 2013, MNRAS, 435, 3481

Woitke P., Helling C., Hunter G. H., Millard J. D., Turner G. E., Worters M., Blecic J., Stock J. W., 2018, A\&A, 614, A1

\section{APPENDIX A: $\chi^{2}$ MAPS OF ALL PLANETS}

\section{WASP-17b}

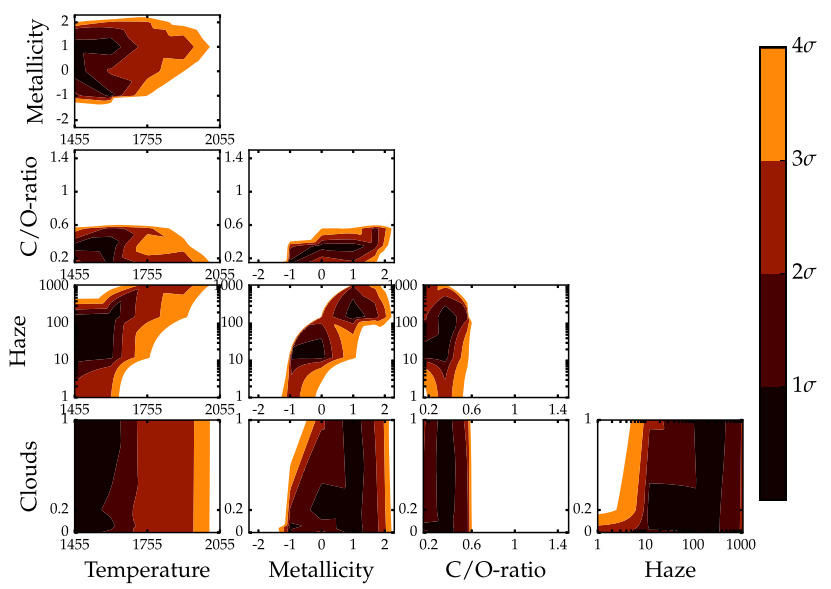

Figure A1. Figure showing WASP-17b $\chi^{2}$ Map, with same format as Fig. 7.

HD-209458b

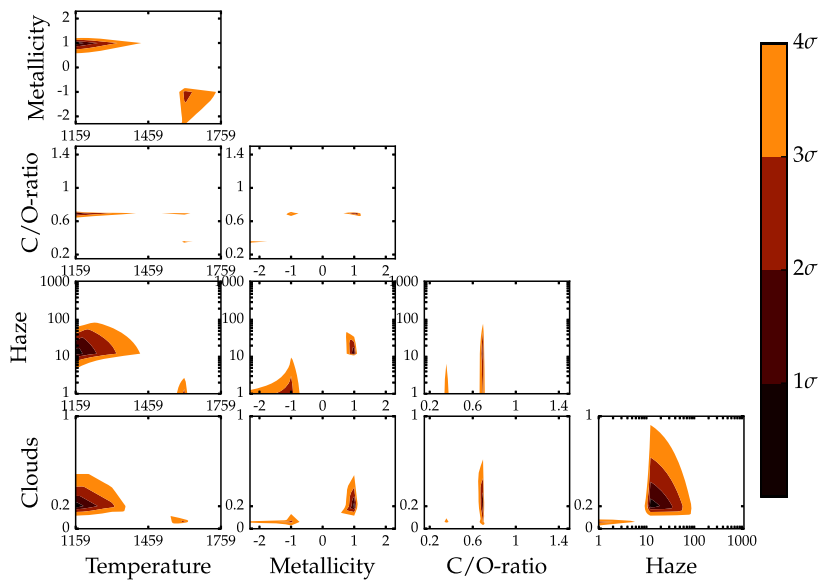

Figure A2. Figure showing HD 209458b $\chi^{2}$ Map, with same format as Fig. 7.
WASP-19b

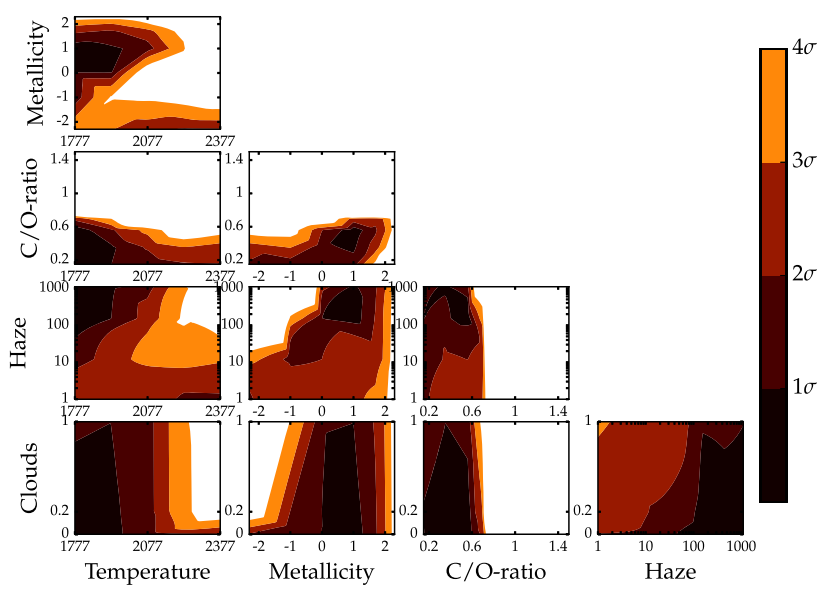

Figure A3. Figure showing WASP-19b $\chi^{2}$ Map, with same format as Fig. 7.

\section{HAT-P-1b}

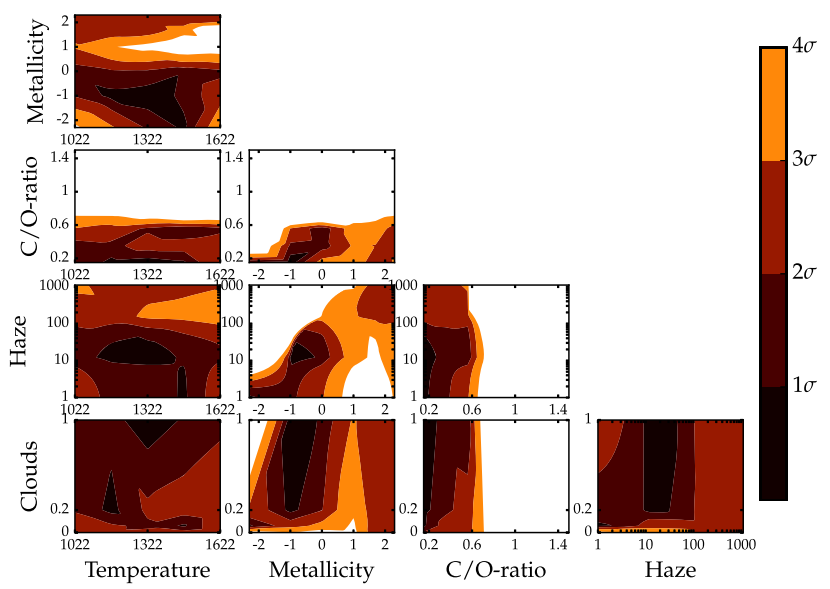

Figure A4. Figure showing HAT-P-1b $\chi^{2}$ Map, with same format as Fig. 7.

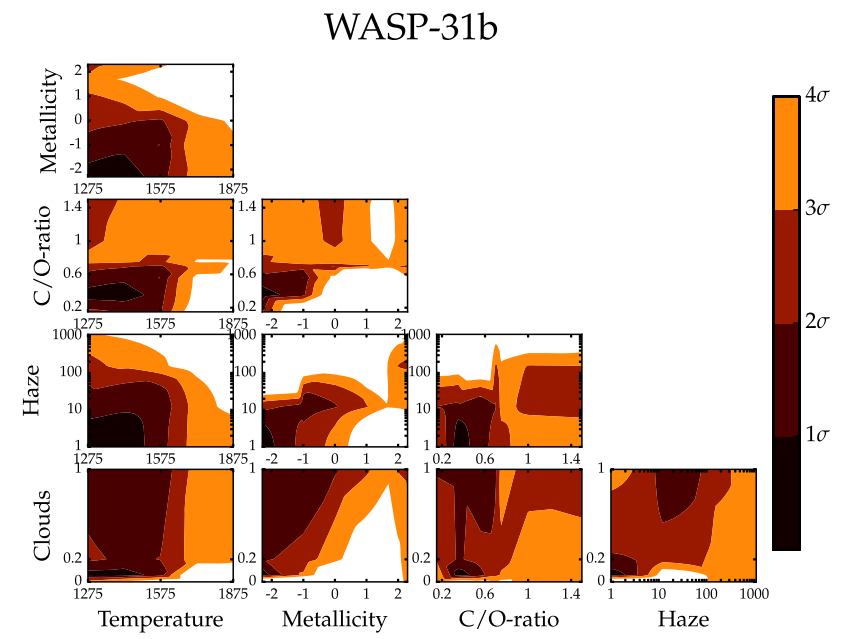

Figure A5. Figure showing WASP-31b $\chi^{2}$ Map, with same format as Fig. 7. 
WASP-12b

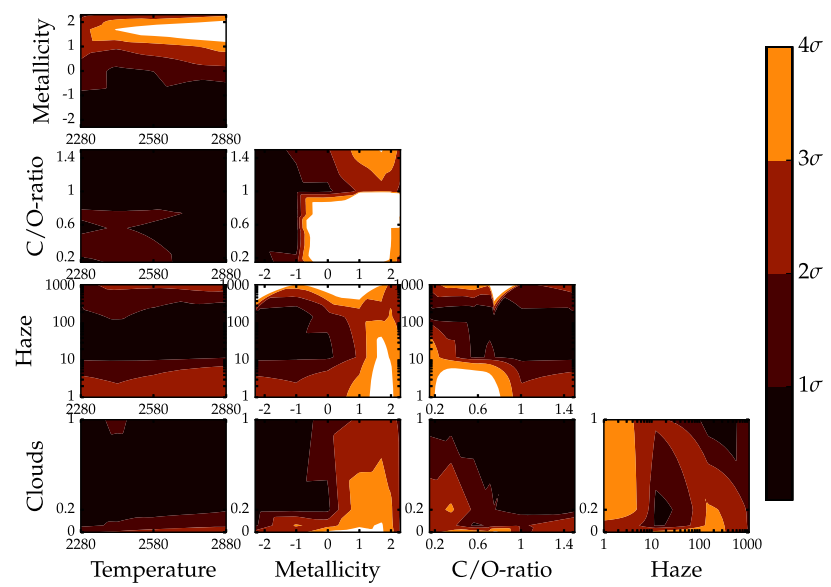

Figure A6. Figure showing WASP-12b $\chi^{2}$ Map, with same format as Fig. 7.

HAT-P-12b

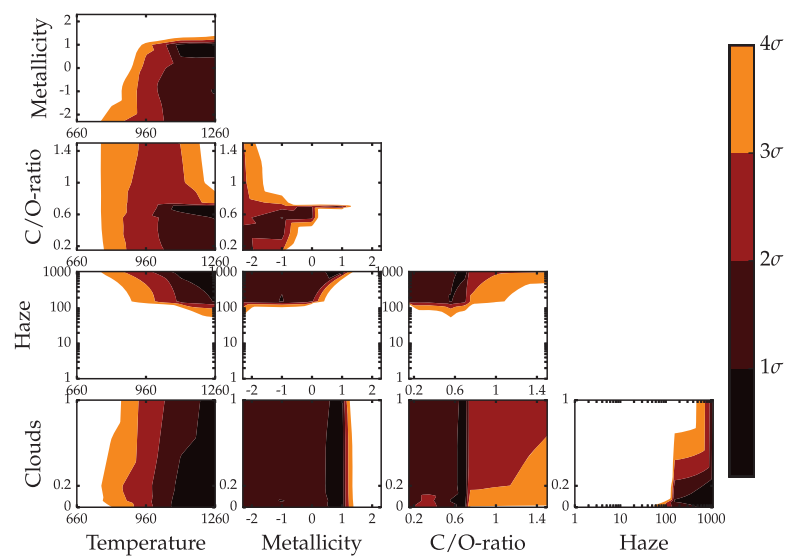

Figure A7. Figure showing HAT-P-12b $\chi^{2}$ Map, with same format as Fig. 7.
HD-189733b

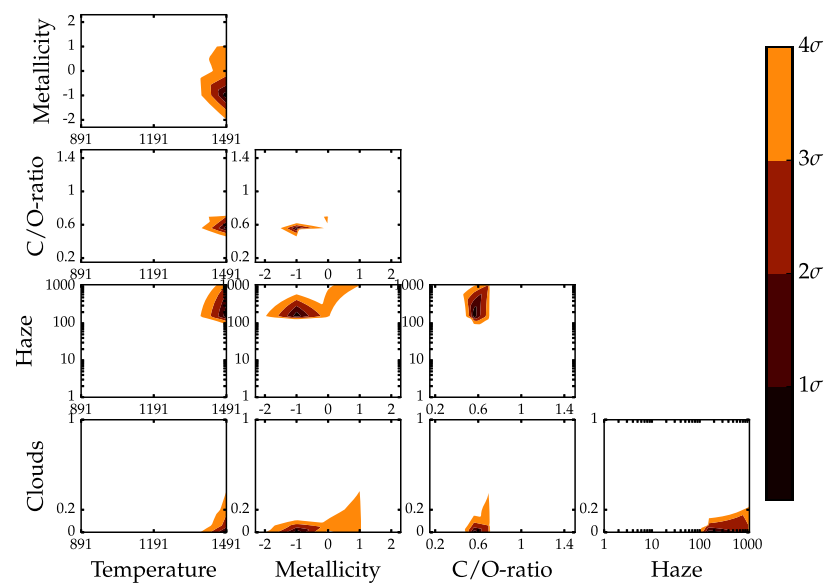

Figure A8. Figure showing HD $189733 b \chi^{2}$ Map, with same format as Fig. 7. 


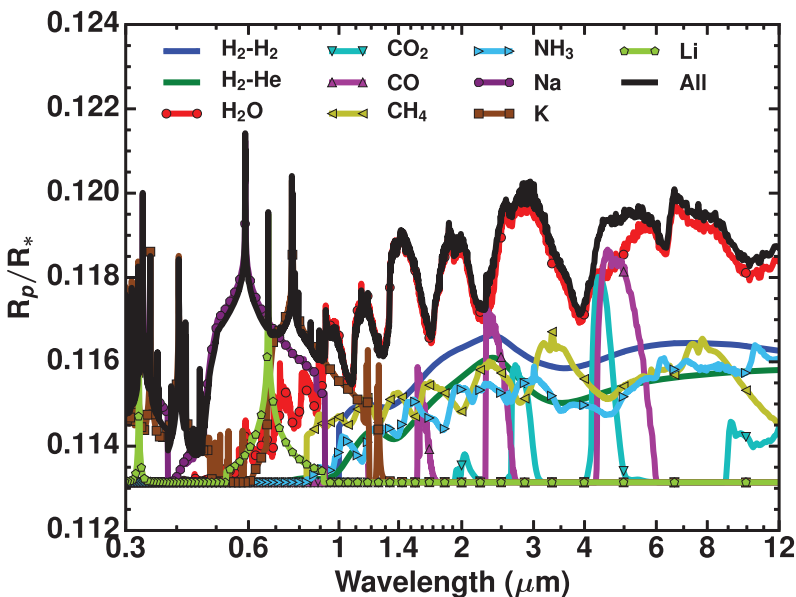

(a)

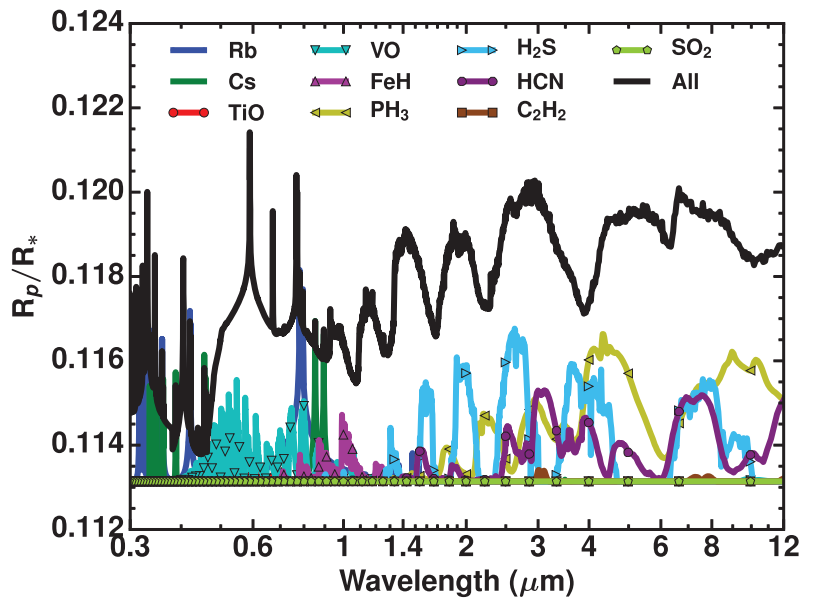

(b)

Figure A10. (a) Figure showing transmission spectra features of each individual molecule used in ATMO (1 to 10 ). $\mathrm{H}_{2}-\mathrm{H}_{2}\left(\right.$ blue), $\mathrm{H}_{2}-\mathrm{He}$ (green), $\mathrm{H}_{2} \mathrm{O}$ (red), $\mathrm{CO}_{2}$ (cyan), $\mathrm{CO}$ (magenta), $\mathrm{CH}_{4}$ (yellow), $\mathrm{NH}_{3}$ (lightblue), $\mathrm{Na}$ (purple), $\mathrm{K}$ (brown), Li (lightgreen) and all 20 opacities (black). (b) Figure showing transmission spectra features of each individual molecule used in ATMO (11 to 20). Rb (blue), Cs (green), TiO (red), $\mathrm{VO}$ (cyan), $\mathrm{FeH}$ (magenta), $\mathrm{PH}_{3}$ (yellow), $\mathrm{H}_{2} \mathrm{~S}$ (lightblue), $\mathrm{HCN}$ (purple), $\mathrm{C}_{2} \mathrm{H}_{2}$ (brown), $\mathrm{SO}_{2}$ (lightgreen) and all 20 opacities (black). No $R_{\mathrm{p}} / R_{\star}$ offset was applied while plotting. Individual simulations are divided into blocks of 10 while plotting for clarity.

This paper has been typeset from a $\mathrm{T}_{\mathrm{E}} \mathrm{X} / \mathrm{L} \mathrm{T} \mathrm{E} \mathrm{X}$ file prepared by the author. 